\title{
Treadmill Exercise Alleviates Circadian Rhythm Disruption- Induced Memory Deficits by Activation of Glucocorticoid Receptor and Brain-Derived Neurotrophic Factor-Dependent Pathway
} \author{
Kyungjin Kim³ ${ }^{3}$ Khae Hawn Kim ${ }^{4}$ \\ ${ }^{1}$ Department of Physiology, College of Medicine, Kyung Hee University, Seoul, Korea \\ ${ }^{2}$ Department of Sport \& Health Science, College of Natural Science, Sangmyung University, Seoul, Korea \\ ${ }^{3}$ Department of Brain \& Cognitive Science, Daegu Gyeongbuk Institute of Science \& Technology, Daegu, Korea \\ ${ }^{4}$ Department of Urology, Gachon University School of Medicine, Gil Medical Center, Incheon, Korea
}

Sung-Eun Kim ${ }^{1}$, Il-Gyu Ko ${ }^{1}$, Eun-Sang Ji ${ }^{1}$, Jun-Jang Jin ${ }^{1}$, Lakkyong Hwang ${ }^{1}$, Sang-Hoon Kim ${ }^{1,2}$, Sehyung Cho $^{1}$, Chang-Ju Kim ${ }^{1}$,

Purpose: Circadian rhythm affects learning process, memory consolidation, and long-term memory. In this study, the alleviating effect of exercise on circadian rhythm disruption-induced memory deficits was investigated.

Methods: BMAL1 knockdown transgenic mice (BMAL1 TG) were used as the BMAL1-TG group and the BMAL1-TG with treadmill exercise group. Female C57BL/6J mice of the same age were used as the wildtype group and the wildtype with treadmill exercise group. The mice in the treadmill exercise groups performed running on a motorized treadmill under the dark-dark conditions for 8 weeks. Short-term memory, nonspatial object memory, and spatial learning memory were determined using stepdown avoidance test, novel object-recognition test, and radial 8-arm maze test. Immunohistochemistry for doublecortin and 5-bromo-2'-deoxyuridine was conducted for the determination of hippocampal neurogenesis. Using the western blot analysis, we determined the expressions of glucocorticoid receptor (GR) and factors related to the neurogenesis and memory consolidation, such as brain-derived neurotrophic factor, tyrosine kinase B, p44/42 mitogen-activated protein kinase, cyclic AMP-responsive element binding protein, phosphatidylinositol 3-kinase, protein kinas $\mathrm{B}$, protein kinase $\mathrm{C}$ alpha, early-growth-response gene 1 .

Results: Circadian rhythm disruption impaired memory function through inhibiting the expressions of GR and the factors related to neurogenesis and memory consolidation. Treadmill exercise improved memory function via enhancing the expressions of GR and above-mentioned factors.

Conclusions: Treadmill exercise acts as the zeitgeber that improves memory function under the circadian rhythm disrupted conditions.

Keywords: BMAL1; Circadian rhythm; Memory impairment; Treadmill exercise

- Research Ethics: The procedure of this study followed the guidelines of Korean Academy of Medical Science and the regulations of National Institutes of Health (NIH) and. We obtained the approval from the Kyung Hee University Institutional Animal Care and Use Committee (Seoul, Korea) (KHUASP [SE]-18-096).

- Conflict of Interest: KHK, an associate editor of INJ, is the corresponding author of this article. However, he played no role whatsoever in the editorial evaluation of this article or the decision to publish it. Except for that, no potential conflict of interest relevant to this article was reported.

\section{- HIGHLIGHTS}

- Circadian rhythm disruption impairs memory function.

- Circadian rhythm disruption inhibits the factors related to neurogenesis and memory consolidation.

- Treadmill exercise improves memory function via enhancing these factors under the circadian rhythm disrupted conditions.

Corresponding author: Khae Hawn Kim (iD http://orcid.org/0000-0002-7045-8004 Department of Urology, Gachon University School of Medicine, Gil Medical Center, 21 Namdong-daero 774beon-gil, Namdong-gu, Incheon 21565, Korea E-mail: kimcho99@gilhospital.com / Tel: +82-32-460-3334 / Fax: +82-32-460-8414 Submitted: January 5, 2019 / Accepted after revision: February 12, 2019
This is an Open Access article distributed under the terms of the Creative Commons Attribution Non-Commercial License (http://creativecommons.org/licenses/by-nc/4.0/) which permits unrestricted non-commercial use, distribution, and reproduction in any medium, provided the original work is properly cited. 


\section{INTRODUCTION}

In mammals, circadian rhythm depends on the integrity of the master circadian oscillator placed in the suprachiasmatic nucleus (SCN) of hypothalamus, which synchronizes peripheral oscillators present in the cells of most organs and tissues [1]. Circadian rhythm affects various physiological functions, such as feeding behavior, regulation of body temperature, and secretion of hormone, thus disruption of circadian rhythm is related to many diseases [1].

Circadian rhythm affects learning process, memory consolidation, and long-term potentiation (LTP) at CA1 in the hippocampal slices [2,3]. Hippocampus exhibits rhythms in clock genes affecting and being affected by central cell signaling pathways, such as mitogen-activated protein kinase (MAPK) and cyclic AMP (cAMP) [4]. The hippocampus gains circadian information via adrenal glucocorticoid [4].

Glucocorticoid is biosynthesized in the adrenal gland and regulates homeostasis, ion transport, glycogenolysis, and immune response [5]. Glucocorticoid modulates synaptic plasticity, neurogenesis, and memory formation in the hippocampus $[3,6]$. Glucocorticoid binds to following 2 main receptors: one is glucocorticoid receptor (GR) and the other is mineralocorticoid receptor (MR). These MR and GR are expressed throughout the brain, at highest level in the hippocampus, however, the functions of these receptors are different. MR modulates the initial phase of memory encoding, whereas, GR is involved in the memory consolidation [5].

Memory consolidation is regulated by a brain-derived neurotrophic factor (BDNF)-dependent autoregulatory loop [7]. BDNF plays an important regulatory role in the survival and differentiation of neurons, and then BDNF is an ideal candidate mediating the process of learning and memory [8]. Aging and exercise affect the expression of BDNF in the hippocampus, leading to promotion or impairment of learning and memory function $[9,10]$. In particular, exercise is known to improve learning and memory function by enhancing BDNF expression in the hippocampus $[11,12]$. CLOCK mutant mice exhibited exercise intolerance and reduced mitochondrial content in skeletal muscle, whereas, endurance exercise increased exercise tolerance and mitochondrial level, suggesting that exercise controls circadian rhythm [13].

Circadian rhythm influences memory acquisition and recall [4]. In the present study, we investigated the alleviating effect of exercise on circadian rhythm disruption-induced memory deficits using BMAL1 knockdown transgenic mice.

\section{MATERIALS AND METHODS}

\section{Animals and Treatments}

BMAL1 knockdown transgenic mice (BMAL1 TG, 9-12 weeks of age) strains were used as the BMAL1-TG group (TG, $n=10$ ) [14] and the BMAL1-TG with treadmill exercise group (TG-EX, $\mathrm{n}=10$ ). Other female C57BL/6J mice of the same age were used as the wildtype group (WT, $n=10$ ) and the wildtype with treadmill exercise group (WT-EX, $\mathrm{n}=10$ ). The mice were kept in constant darkness for 8 weeks. The mice in the treadmill exercise groups performed running on a motorized treadmill for 30 minutes daily, 5 days per a week, for 8 consecutive weeks, under the dark-dark conditions. The exercise load was running at a 3 $\mathrm{m} / \mathrm{min}$ for 5 minutes, $5 \mathrm{~m} / \mathrm{min}$ for 5 minutes, and $8 \mathrm{~m} / \mathrm{min}$ for 20 minutes, with $0^{\circ}$ inclination.

\section{Step-Down Avoidance Test}

According to the previously described method [15], the latency of the step-down avoidance test was measured for the evaluation of short-term memory. The mice were trained in a stepdown avoidance test, and then, the latency (second) was measured at 2 hours after training session. The mice were placed on a platform with width, length, and height of $25 \mathrm{~cm} \times 7 \mathrm{~cm} \times 2.5$ $\mathrm{cm}$. The platform has a parallel stainless steel bars measuring 25 $\mathrm{cm} \times 42 \mathrm{~cm}$ with a diameter of $0.1 \mathrm{~cm}$ and a spacing of $1 \mathrm{~cm}$. During the training session, $0.5 \mathrm{~mA}$ scramble foot shock was applied to the mice for 2 seconds immediately after stepping down. We defined the time interval as the latency until the mouse came down and reached four feet on the grid. The latency of 300 seconds or longer was calculated as 300 seconds.

\section{Novel Object Recognition Test}

For the assessment of non-spatial object memory, we conducted the novel object-recognition test using an open field box with width, length, and height of $45 \mathrm{~cm} \times 45 \mathrm{~cm} \times 30 \mathrm{~cm}$, as previously described method [16]. Before to test, the mice were accustomed to the box for 15 minutes without objects. On the object trial, the mice were placed into the test box with 2 identical objects (approximately $30 \mathrm{~cm}$ apart from each other), and then allowed to explore for 10 minutes. After 1 hour waiting time, the mice were put back into the test box and allowed to explore the objects. One object was the same as that used in the previous training session, and the other one was a novel object. We measured the time spent for exploring novel object and familiar object. They were considered to be exploring when they were facing, sniffing, or bit- 
ing the object within a $1.5-\mathrm{cm}$ radius. This test was conducted under the dark conditions, and the test box and objects were washed using $70 \%$ ethanol between tests. The percentage of novel object recognition time and the discrimination ratio were obtained by the following formula. Novel object recognition time $(\%)=($ novel object time/[novel object time+familiar object time] )$\times 100$. Discrimination ratio $=($ novel object time-familiar object time)/(novel object time+familiar object time).

\section{Radial 8-Arm Maze Test}

As the previously described method [17], we performed radial 8 -arm maze test for the determination of spatial learning memory, using a radial 8 -arm maze apparatus. This apparatus is seated $1 \mathrm{~m}$ above the floor and it has a central octagonal plate (30 $\mathrm{cm}$ in diameter) with 8 radiating arms $(50 \mathrm{~cm}$ in length, 10 $\mathrm{cm}$ in width). At the end of the arm, there was a small dish filled with water ( $3 \mathrm{~cm}$ in diameter, $1 \mathrm{~cm}$ in depth). Before the spatial learning memory test, the mice were trained 3 times. For training sessions, the water supply to the mice was stopped for 24 hours. After each training session, water was supplied to the mice for 5 minutes. During the radial 8-arm maze test, the spending time for seeking water was calculated as the latency. This test was stopped when the mice drink water in all 8 arms or experiment time was over 8 minutes. When the mice entered arm that was already visited, it was counted as the error number. The continuous number of correct choices before the first error occurred was counted as the correct number.

\section{Tissue Preparation for Immunohistochemistry}

Immediately after finishing the behavior testing, the mice were fully anesthetized by Zoletil $50(10 \mathrm{mg} / \mathrm{kg}$, intraperitoneally; Vibac Laboratories, Carros, France). Then, 50mM phosphatebuffered saline (PBS) was transcardially perfused, and the mice were fixed using $4 \%$ paraformaldehyde in $100 \mathrm{mM}$ phosphate buffer $(\mathrm{pH}, 7.4)$. After dissecting the brains, we made $5-\mu \mathrm{m}$ thick coronal sections using a freezing microtome (Leica, Nussloch, Germany).

\section{Doublecortin Immunohistochemistry}

We conducted doublecortin (DCX) immunohistochemistry, as the previously described method [18]. After washing the sections by $0.1 \mathrm{M} \mathrm{PBS}(\mathrm{pH}, 7.4)$, the sections were treated with a $3 \%$ $\mathrm{H}_{2} \mathrm{O}_{2}$ and $20 \%$ methanol. The sections were treated with anti- $\alpha$ doublecortin antibody ( $1 \mu \mathrm{g} / \mathrm{mL}$; Santa Cruz Biotechnology, Santa Cruz, CA, USA) at $4^{\circ} \mathrm{C}$ for 48 hours, then the sections were incubated with donkey a-goat biotinylated antibody for 1 hour at room temperature (Chemicon Inc., Temecula, CA, USA). The sections were treated with $\mathrm{ABC}$ Elite kit (Vector Laboratories, Burlingame, CA, USA) for 1 hour. After that, $0.02 \%$ diaminobenzidine (DAB) with $0.03 \% \mathrm{H}_{2} \mathrm{O}_{2}$ was treated to the sections for 5 minutes. And then, gelatin-coated slides were used for the mounting of the sections.

\section{5-Bromo-2'-Deoxyuridine Immunohistochemistry}

The mice received $50 \mathrm{mg} / \mathrm{kg}$ 5-Bromo-2'-Deoxyuridine (BrdU) (Sigma Chemical Co., St. Louis, MO, USA) intraperitoneally at the third, fourth, tenth, and eleventh day after onset of exercise. We conducted BrdU-specific immunohistochemistry, as the previously described method [18]. After permeabiliztion of the sections with $0.5 \%$ Triton X-100 in PBS for 20 minutes, the section was treated with $50 \%$ formamide- $2 \mathrm{x}$ standard saline citrate at $65^{\circ} \mathrm{C}$ for 2 hours. The sections were denatured using 2 $\mathrm{N} \mathrm{HCl}$ at $37^{\circ} \mathrm{C}$ for 30 minutes, and then washed 2 times by $100 \mathrm{mM}$ sodium borate $(\mathrm{pH}, 8.5)$. BrdU-specific mouse monoclonal antibody (1:600) (Roche, Mannheim, Germany) was treated to the sections during overnight at $4^{\circ} \mathrm{C}$. After incubating the sections with biotinylated mouse secondary antibody (1:200) (Vector Laboratories) for 1 hour, an avidin-peroxidase complex (1:100) (Vector Laboratories) was treated to the sections for next 1 hour. After that, the sections were treated with $50 \mathrm{mM}$ Tris- $\mathrm{HCl}(\mathrm{pH}, 7.6)$ including $0.03 \% \mathrm{DAB}, 40-\mathrm{mg} / \mathrm{mL}$ nickel chloride, and $0.03 \% \mathrm{H}_{2} \mathrm{O}_{2}$ for 5 minutes.

Mouse antineuronal nuclei antibody (Chemicon Inc.) was used to detect the differentiation of BrdU-positive cells in the same sections. After incubation of the sections with a biotinylated anti-mouse secondary antibody for 1 hour, reaction mixture composing of $0.03 \% \mathrm{DAB}$ and $0.03 \% \mathrm{H}_{2} \mathrm{O}_{2}$ was treated to the sections for 5 minutes. And then, gelatin-coated slides were used for the mounting of the sections.

\section{Western Blot Analysis}

We performed western blot analysis, as the previously described method [15,17]. After homogenizing hippocampal tissues on ice, hippocampal tissues were lysed using a lysis buffer including $1 \mathrm{mM}$ sodium orthovanadate, 50mM HEPES ( $\mathrm{pH}, 7.5$ ), 10\% glycerol, $1 \%$ Triton X-100, 100mM sodium fluoride, $150 \mathrm{mM}$ $\mathrm{NaCl}, 1.5 \mathrm{mM} \mathrm{MgCl} 6 \mathrm{H}_{2} \mathrm{O}, 1 \mathrm{mM}$ phenylmethylsulfonyl fluoride, and $1 \mathrm{mM}$ ethylene glycol tetraacetic acid. After separating protein $(20 \mu \mathrm{g})$ from the sodium dodecyl sulfate-polyacrylamide gels, the protein was transferred to the nitrocellulose 
membrane. For the primary antibodies, we used rabbit GAPDH antibody (AbFrontier, Seoul, Korea; 1:5,000), rabbit GR antibody (Santa Cruz Biotechnology; 1:1,000), rabbit BDNF antibody (Santa Cruz Biotechnology; 1:1,000), rabbit tyrosine kinase B (TrkB) antibody (Santa Cruz Biotechnology; 1:1,000), rabbit cAMP-responsive element binding protein (CREB) antibody (Santa Cruz Biotechnology; 1:1,000), rabbit phospho-CREB (pCREB) (Ser133) antibody (Santa Cruz Biotechnology; 1:1,000), mouse phosphatidylinositol 3-kinase (PI 3-kinase) p85 $\alpha$ (Santa Cruz Biotechnology; 1:1,000), rabbit phospho-PI 3-kinase p85a (p-PI 3-kinase p85a) (Tyr467) antibody (Santa Cruz Biotechnology; 1:1,000), rabbit protein kinase $\mathrm{C}$ alpha (PKCa) antibody (Santa Cruz Biotechnology; 1:1,000), rabbit phospho-PKCa (p-PKCa) (Ser657) antibody (Merck Millipore Corp., Darmstadt, Germany; 1:1,000), rabbit protein kinas B (Akt) antibody
(Cell Signaling Technology, Beverly, MA, USA; 1:1,000), rabbit phospho-Akt (p-Akt) (Ser473) antibody (Cell Signaling Technology; 1:1,000), rabbit early-growth-response gene 1 (Egr-1) antibody (Santa Cruz Biotechnology; 1:1,000), rabbit total p44/42 MAPK (Erk1/2) antibody (Cell Signaling Technology; 1:1,000), rabbit phospho-p44/42 MAPK (p-Erk1/2) antibody (Cell Signaling Technology; 1:1,000). For the secondary antibodies, we used horseradish peroxidase-conjugated anti-rabbit antibody (Vector Laboratories; 1:2,000) for GAPDH, GR, BDNF, TrkB, Akt, p-Akt, CREB, p-CREB, p-PI 3-kinase p85a, PKCa, p-PKCa, Egr-1, ERK1/2, p-ERK1/2, and horseradish peroxidaseconjugated anti-mouse antibody for PI 3-kinase p85a (Amersham Pharmacia Biothech GmbH, Freiburg, Germany; 1:2,000). An enhanced chemiluminescence detection kit (Santa Cruz Biotechnology) was used to calculate the detected bands.

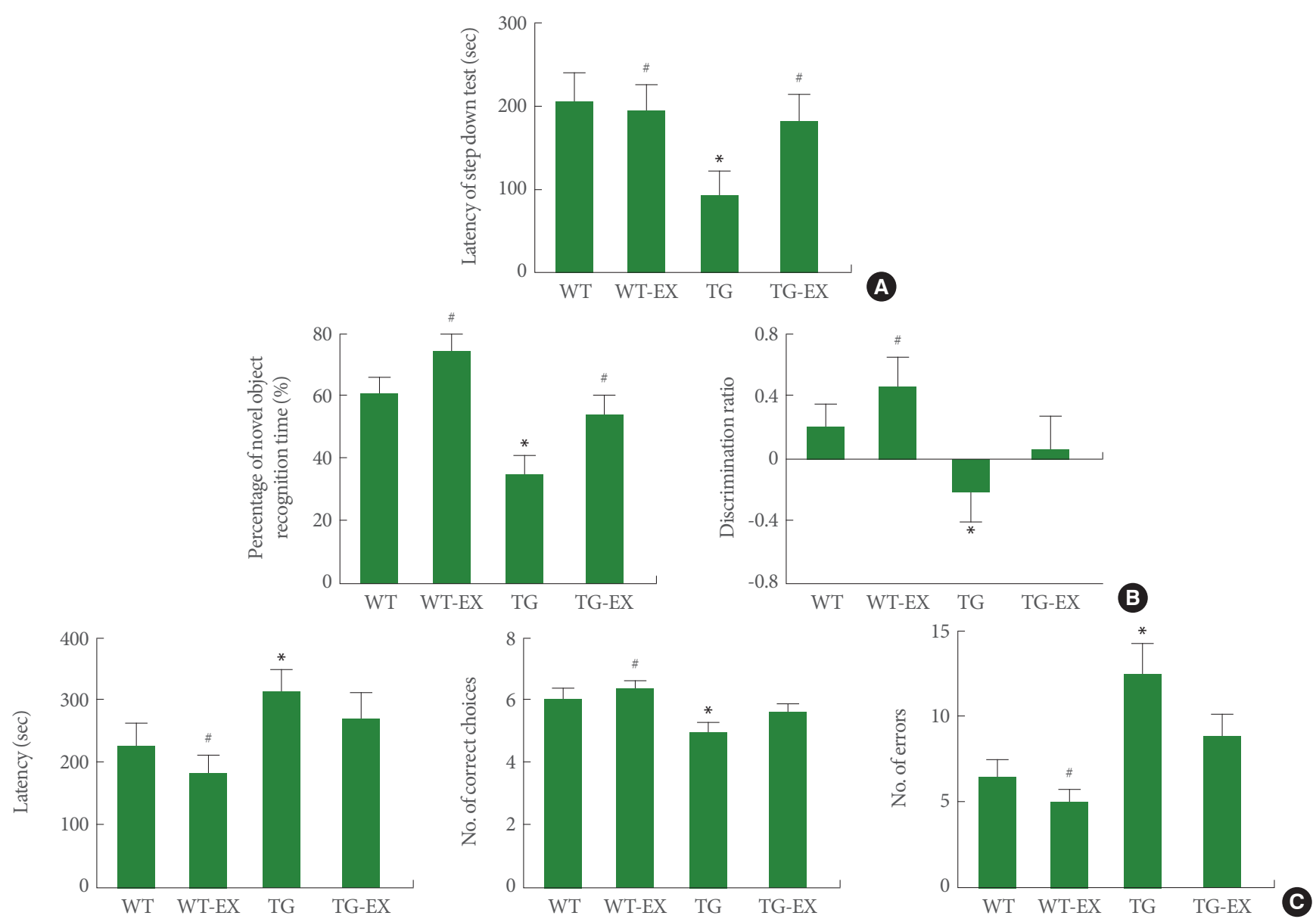

Fig. 1. Effects of treadmill exercise on the performance of behavior tests. (A) Step-down avoidance test. (B) Novel object recognition test. (C) Radial 8-arm maze test. WT, wildtype group; WT-EX, wildtype with treadmill exercise group; TG, BMAL1-transgenic group; TG-EX, BMAL1-transgenic with treadmill exercise group. Data are represented as mean \pm standard error of the mean. ${ }^{*} \mathrm{P}<0.05 \mathrm{com}-$ pared to the WT. ${ }^{*} \mathrm{P}<0.05$ compared to the TG. 


\section{Data Analysis}

Molecular AnalystTM, version 1.4.1 (Bio-Rad, Hercules, CA, USA) was used for the densitometrical calculation of these bands. The numbers of DCX-positive and BrdU-positive cells in the hippocampal dentate gyrus were hemilaterally counted, and the results were expressed as the number of DCX-positive or BrdU-positive cells per $\mathrm{mm}^{2}$ in the hippocampal dentate gyrus. We used the Image-Pro Plus image analysis system (Media Cyberbetics Inc., Silver Spring, MD, USA) to measure the total area of the hippocampal dentate gyrus.

The results were statistically analyzed by Duncan post hoc test following 1-way analysis of variance. Data are presented as the mean \pm standard error of the mean. Statistical significance was considered when P-value was less than 0.05 .

\section{RESULTS}

\section{Memory Function in Circadian Rhythm Disturbed Mice}

For the step-down avoidance test, disruption of circadian rhythm showed shorter latency $(\mathrm{P}<0.05)$. On the contrary, treadmill exercise lengthened this latency in TG mice $(P<0.05)$ (Fig. 1A). For the novel object recognition test, disruption of circadian rhythm showed lower recognition time percentage and discrimination ratio $(\mathrm{P}<0.05)$. On the contrary, treadmill exercise increased the percentage of recognition time in TG mice $(\mathrm{P}<0.05)$ (Fig. 1B). For the radial arm maze test, disruption of circadian rhythm showed longer time, more error, and less correct during the task $(\mathrm{P}<0.05)$. However, treadmill exercise showed no significant effect on latency, number of error, and number of correct (Fig. 1C).

\section{GR Expression in Circadian Rhythm Disturbed Mice}

Disruption of circadian rhythm inhibited the expression of GR in the hippocampus $(\mathrm{P}<0.05)$. On the contrary, treadmill exercise improved the expression of GR both in TG mice and WT mice $(\mathrm{P}<0.05)$ (Fig. 2A).

\section{BDNF and TrkB Expressions in Circadian Disturbed Mice}

Disruption of circadian rhythm reduced the expressions of BDNF and TrkB in the hippocampus $(\mathrm{P}<0.05)$. On the contrary, treadmill exercise improved the expressions of BDNF and TrkB both in TG mice and WT mice $(\mathrm{P}<0.05)$ (Fig. 2B).

\section{p-Erk1/2 Expression in Circadian Rhythm Disturbed Mice}

Disruption of circadian rhythm reduced the ratio of $\mathrm{p}$-Erk $1 / 2$ to Erk1/2 in the hippocampus $(\mathrm{P}<0.05)$. On the contrary, treadmill exercise improved the expression of $\mathrm{p}$-Erk1/2 adjusted by Erk1/2 both in TG mice and WT mice $(\mathrm{P}<0.05)$ (Fig. 3A).
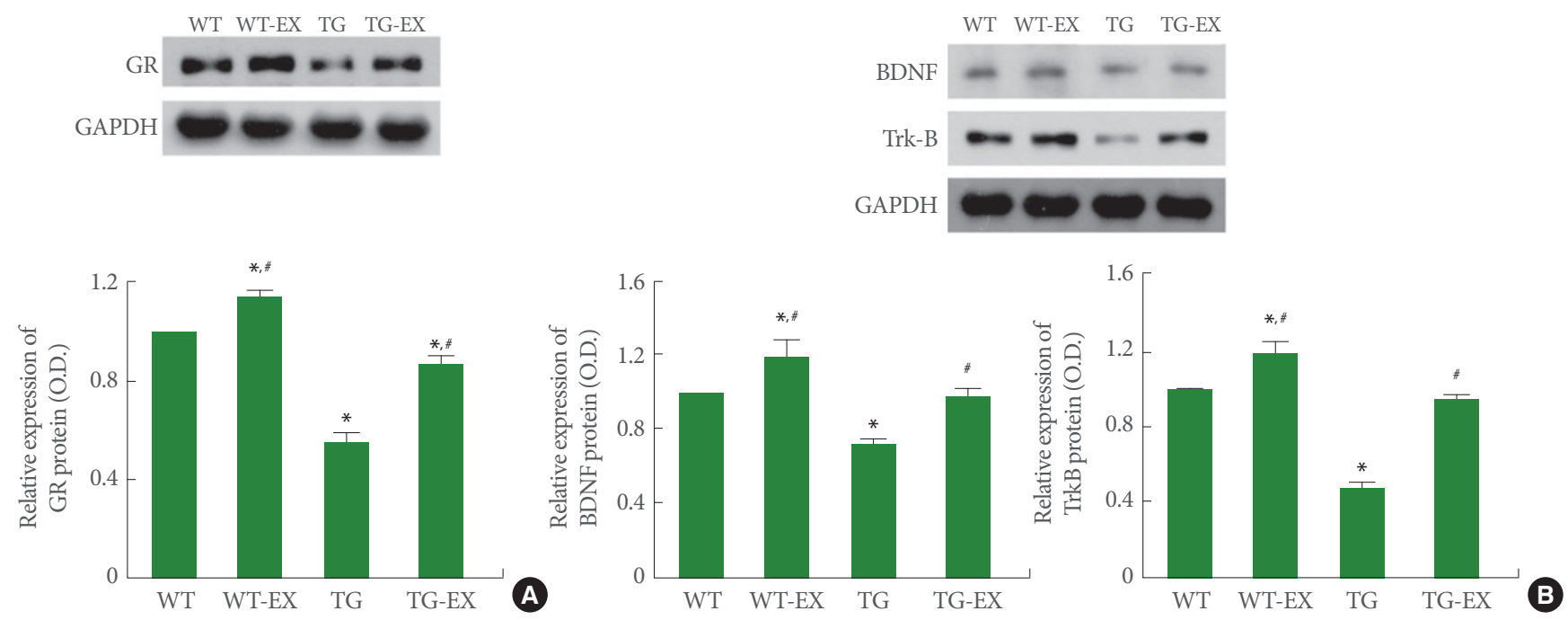

Fig. 2. Effects of treadmill exercise on the expressions of glucocorticoid receptor (GR), brain-derived neurotrophic factor (BDNF), and tyrosine kinase B (TrkB). (A) GR expression in the hippocampus. (B) BDNF and TrkB expressions in the hippocampus. WT, wildtype group; WT-EX, wildtype with treadmill exercise group; TG, BMAL1-transgenic group; TG-EX, BMAL1-transgenic with treadmill exercise group. Data are represented as mean \pm standard error of the mean. ${ }^{*} \mathrm{P}<0.05$ compared to the WT. ${ }^{*} \mathrm{P}<0.05$ compared to the TG. 


\section{p-CREB Expression in Circadian Rhythm Disturbed Mice}

Disruption of circadian rhythm reduced the ratio of $\mathrm{p}$-CREB to CREB in the hippocampus $(\mathrm{P}<0.05)$. On the contrary, treadmill exercise improved the expression of $\mathrm{p}$-CREB adjusted by CREB both in TG mice and WT mice $(\mathrm{P}<0.05)$ (Fig. 3B).

\section{p-PI 3-Kinase p85a Expression in Circadian Rhythm Disturbed Mice}

Disruption of circadian rhythm reduced the ratio of p-PI $3 \mathrm{ki}-$ nase $\mathrm{p} 85 a$ to PI 3 kinase $\mathrm{p} 85 a$ in the hippocampus $(\mathrm{P}<0.05)$.
On the contrary, treadmill exercise improved the expression of p-PI 3 kinase p85a adjusted by PI 3 kinase p85 $\alpha$ in TG mice $(\mathrm{P}<0.05)$ (Fig. 3C).

\section{p-Akt Expression in Circadian Rhythm Disturbed Mice}

Disruption of circadian rhythm reduced the ratio of p-Akt to Akt in the hippocampus $(\mathrm{P}<0.05)$. On the contrary, treadmill exercise improved the expression of $\mathrm{p}$-Akt adjusted by Akt both in TG mice and WT mice $(\mathrm{P}<0.05)$ (Fig. 3D).
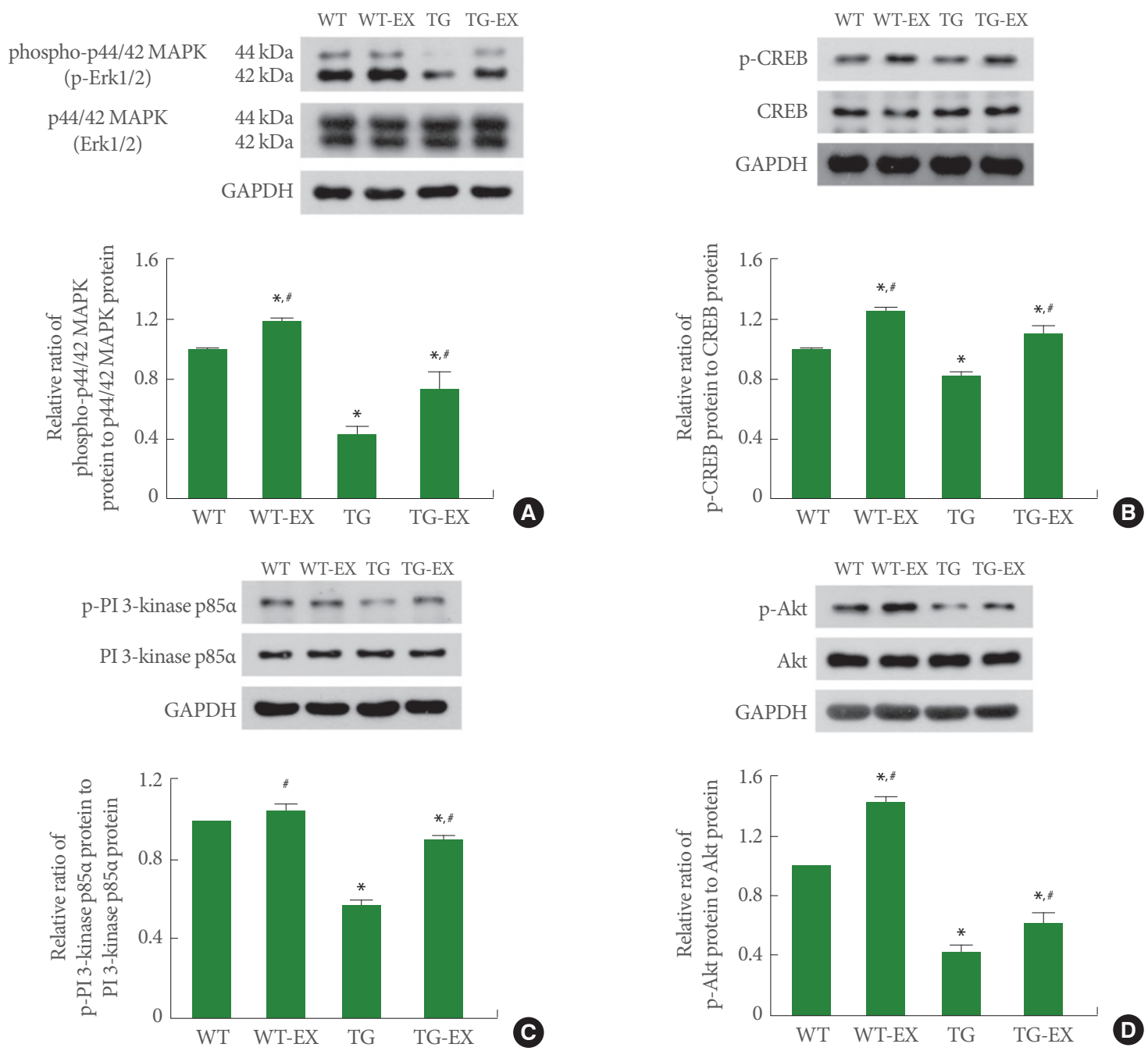

Fig. 3. Effects of treadmill exercise on the expressions of mitogen-activated protein kinase (MAPK), cAMP-responsive element binding protein (CREB), phosphatidylinositol 3-kinase (PI 3-kinase), and protein kinas B (Akt). (A) p-p44/42 MAPK (p-Erk1/2) expression in the hippocampus. (B) p-CREB expression in the hippocampus. (C) p-PI 3-kinase p85a expression in the hippocampus. (D) pAkt expression in the hippocampus. WT, wildtype group; WT-EX, wildtype with treadmill exercise group; TG, BMAL1-transgenic group; TG-EX, BMAL1-transgenic with treadmill exercise group. Data are represented as mean \pm standard error of the mean. ${ }^{*} \mathrm{P}<0.05$ compared to the $\mathrm{WT}$. ${ }^{*} \mathrm{P}<0.05$ compared to the TG. 

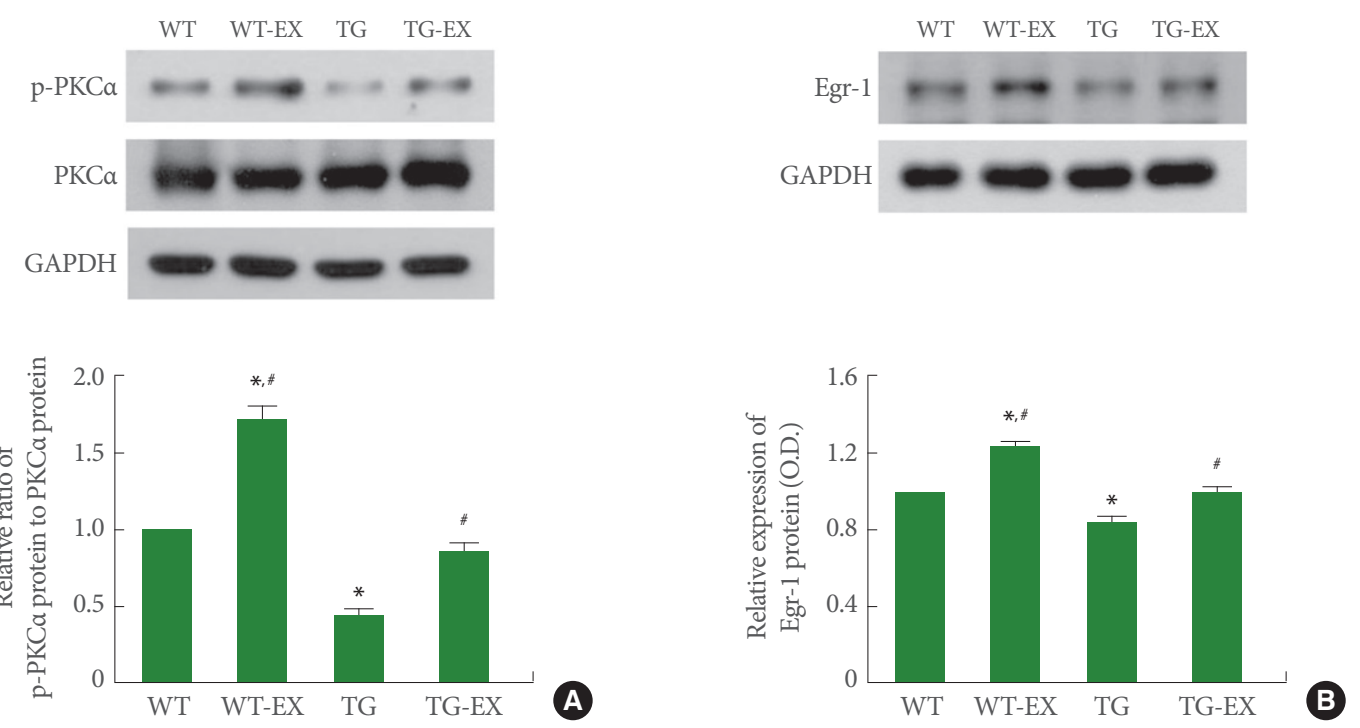

Fig. 4. Effects of treadmill exercise on the expressions of protein kinase $\mathrm{C}$ (PKC) and Egr-1. (A) p-PKCa expression in the hippocampus. (B) Egr-1 expression in the hippocampus. WT, wildtype group; WT-EX, wildtype with treadmill exercise group; TG, BMAL1transgenic group; TG-EX, BMAL1-transgenic with treadmill exercise group. Data are represented as mean \pm standard error of the mean. ${ }^{*} \mathrm{P}<0.05$ compared to the $\mathrm{WT}$. ${ }^{\#} \mathrm{P}<0.05$ compared to the TG.

\section{p-PKCa Expression in Circadian Rhythm Disturbed Mice}

Disruption of circadian rhythm reduced the ratio of p-PKCa to $\mathrm{PKC} \alpha$ in the hippocampus $(\mathrm{P}<0.05)$. On the contrary, treadmill exercise improved the expression of $\mathrm{p}$-PKCa adjusted by PKCa both in TG mice and WT mice $(\mathrm{P}<0.05)$ (Fig. 4A).

\section{Egr-1 Expression in Circadian Rhythm Disturbed Mice}

Disruption of circadian rhythm inhibited the expression of Egr1 in the hippocampus $(\mathrm{P}<0.05)$. On the contrary, treadmill exercise improved Egr-1 expression both in TG mice and WT mice $(\mathrm{P}<0.05)$ (Fig. 4B).

\section{Neurogenesis in Circadian Rhythm Disturbed Mice}

Disruption of circadian rhythm reduced the number of DCXpositive cells in the hippocampal dentate gyrus $(\mathrm{P}<0.05)$. On the contrary, treadmill exercise enhanced the number of DCXpositive cells both in TG mice and WT mice $(\mathrm{P}<0.05)$ (Fig. 5A).

Disruption of circadian rhythm reduced the number of BrdUpositive cells in the hippocampal dentate gyrus $(\mathrm{P}<0.05)$. On the contrary, treadmill exercise enhanced the number of BrdU-positive cells both in TG mice and WT mice $(\mathrm{P}<0.05)$ (Fig. 5B).

\section{DISCUSSION}

As SCN is the anatomical center for generation of circadian rhythm, SCN lesions interfere with hippocampus-dependent memory [19]. Mice lacking forebrain BMAL1 showed deficits in the spatial and novel object location memory [20]. In this study, BMAL1-knockdown mice displayed deficits of shortterm, non-spatial object, and spatial learning memory. In contrast, treadmill exercise showed alleviating effect on deficits of short-term and non-spatial object memory (Fig. 1).

BMAL1-knockout mice exhibited impairment of spatial learning memory with suppressed MAPK activation [21]. MAPK activation stimulates CREB pathway necessary for spatial and social learning [19]. CREB plays a critical role in memory formation and integration through modulating adult hippocampal neurogenesis [22]. In this study, Erk1/2 phosphorylation in the hippocampus of circadian rhythm disturbed mice was weakened compared with wildtype mice (Fig. 3A). CREB activation induced by phosphorylation at a serine residue (Ser 133) was also decreased in the hippocampus of circadian rhythm disturbed mice (Fig. 3B).

$\mathrm{BMAL1}^{-1-}$ mice show reduced glucocorticoid level under the both light-dark and dark-dark conditions without change of ACTH secretion [23]. GR expression in $\mathrm{BMAL1}^{-1-}$ mouse embryonic fibroblasts was reduced compared to the wildtype mouse embryonic fibroblasts [1]. In this study, GR expression in the hippocampus of BMAL1-knockdown mice under the constant darkness was decreased compared with WT mice. 

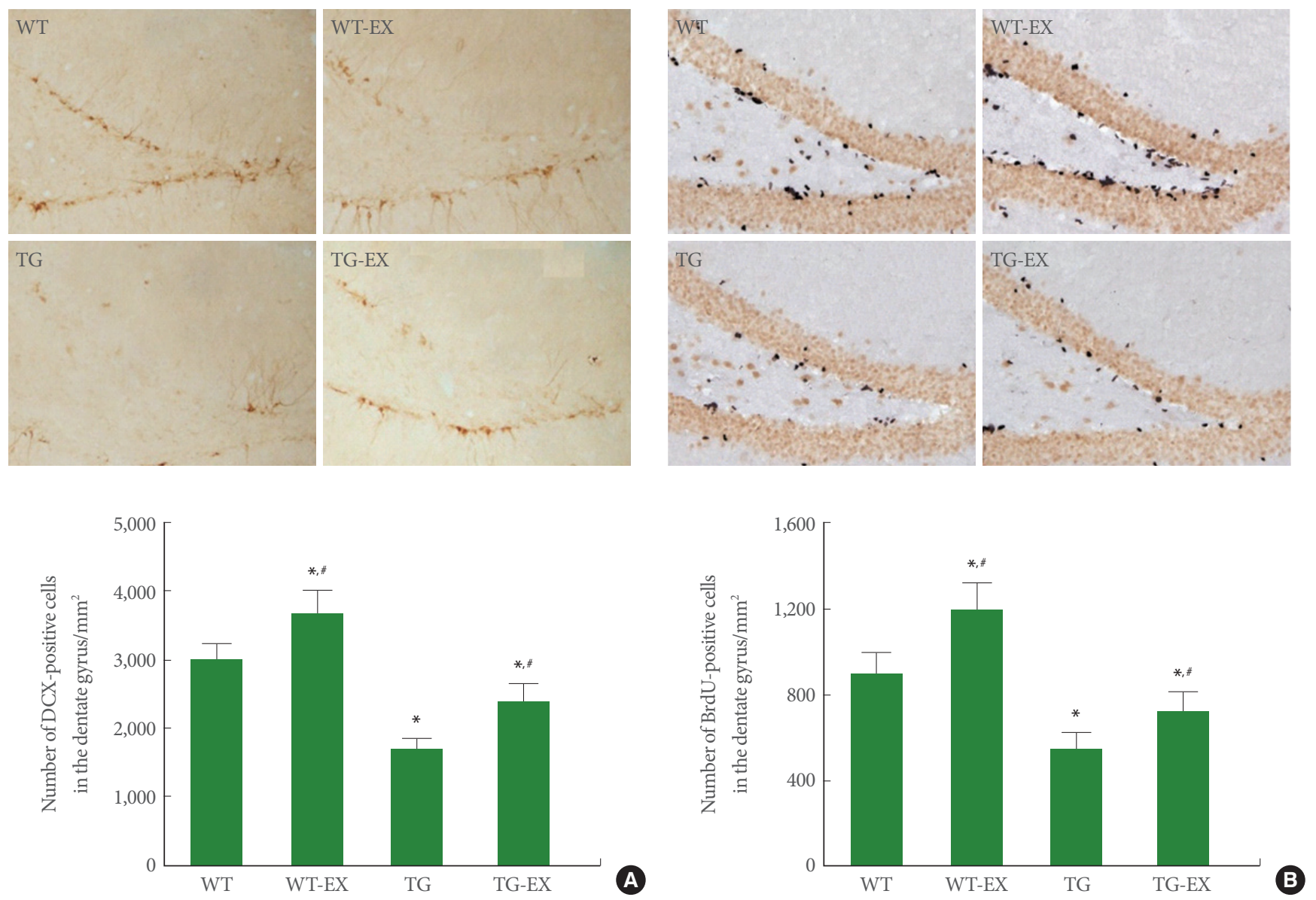

Fig. 5. Effects of treadmill exercise on cell proliferation and neurogenesis in the hippocampal dentate gyrus. (A) Doublecortin (DCX)positive cells in the hippocampal dentate gyrus. Upper panel: Photomicrographs of DCX-positive cells. The scale bar represents 50 $\mu \mathrm{m}$. Lower panel: The number of DCX-positive cells in each group. (B) 5-Bromo-2'-deoxyuridine (BrdU)-positive cells in the hippocampal dentate gyrus. Upper panel: Photomicrographs of BrdU-positive cells. The scale bar represents $50 \mu \mathrm{m}$. Lower panel: The number of BrdU-positive cells in each group. WT, wildtype group; WT-EX, wildtype with treadmill exercise group; TG, BMAL1-transgenic group; TG-EX, BMAL1-transgenic with treadmill exercise group. The data are represented as mean \pm standard error of the mean. ${ }^{*} \mathrm{P}<0.05$ compared to the WT. ${ }^{*} \mathrm{P}<0.05$ compared to the TG.

Treadmill exercise enhanced the expression of GR in the hippocampus of circadian rhythm disturbed mice (Fig. 2A).

GR is activated by binding to glucocorticoid hormone, and translocated to cell nuclei, then affects transcription through binding to glucocorticoid responsive element (GRE) in the promoter region of the target genes. During period of inactivity of the circadian cycle, low level of glucocorticoid results in a marked decrease in nuclear GR and GRE binding level [24]. Injection of GR antagonist into the dorsal hippocampus impaired long-term avoidance memory in rats through suppressing phospho-calcium calmodulin kinase II a, p-Akt, p-CREB, p-TrkB, p-ERK, and phospho-phospholipase $\mathrm{C} \gamma$ [7]. For the memory consolidation, BDNF-dependent pathway is an important downstream effector of GR activation [5]. In this study, circadian rhythm disturbed mice showed reduced expressions of $\mathrm{p}-\mathrm{CREB}, \mathrm{BDNF}$, TrkB, p-PI3 kinase p-85a, p-Akt, and p-Erk1/2 in the hippocampus with impaired memory function. Treadmill exercise increased the expressions of BDNF, TrkB, and facilitated the phosphorylation of CREB, PI3 kinase p85a, Akt, Erk1/2 in the hippocampus of circadian rhythm disturbed mice, contributing to improvement of memory function (Figs. 1-3).

Hippocampal BDNF mediates the exercise-induced enhancing effect on synaptic plasticity and cognition [11,12]. CREB potentiates consolidation and performance of memory through regulation of BDNF expression [25]. The beneficial effect of exercise on learning ability and memory function is due to activation of 
CREB and Akt in the hippocampus by exercise, which in turn culminates in the increase of BDNF [9]. Treadmill exercise improves spatial learning memory via increased phosphorylation of PI3K, Akt, and Erk1/2 in the hippocampus of autistic rats [26].

TrkB activates the PLC $\gamma$, which in turn, produces diacylglycerol (DAG) and inositol 1,4,5-trisphosphate (IP3) [27]. In particular, DAG activates PKC, and PKC activity plays a crucial role in diverse memories and cognitive function [27]. Especially, PKCa modulates the process of differentiation and regeneration of neurons, and PKCa is associated with the acquisition of learning [28]. Lin et al. [29] also suggested that running exercise improves learning ability and memory function against $\mathrm{Al}-$ zheimer disease through enhancing expressions of p-PKC, $\mathrm{p}$ TrkB, and p-Akt. In this study, treadmill exercise activated $\mathrm{PKC} \alpha$ in the hippocampus of circadian rhythm disturbed mice, showing that decreased PKCa activity is associated with impaired memory function (Figs. 1, 4A).

Activation of hippocampal GR increases Egr-1 expression through enhancing enzymatic activity in the MAPK signaling pathway including phosphorylation of CREB [30]. Since Egr-1 is required for late LTP and long-term memory [31], Egr1-knockout mice showed impairment of long-term memory [32]. In this study, treadmill exercise enhanced Egr-1 expression in the hippocampus of both TG mice and wildtype mice (Fig. 4B), which is consistent with increased expressions of BDNF, p-CREB, and p-Erk1/2 (Figs. 2B, 3A, and 3B).

BDNF and phosphorylated CREB promote new cell formation in the hippocampal dentate gyrus, and then contribute to memory consolidation and memory improvement [22,33]. BDNF promotes birth, survival, and maturation of new neurons in the hippocampus [33]. Phosphorylated CREB is associated with newly born neurons, such areas as hippocampal dentate gyrus or subventricular zone [22]. Treadmill exercise is known to increase the production of new neurons by increasing BDNF expression in the hippocampus, as a result, it achieves learning and memory enhancement $[10,11]$. In this study, consistent with reduced expressions of BDNF, TrkB, p-CREB, and p-Erk1/2 in the hippocampus of BMAL1-knockdown mice, circadian rhythm disturbed mice showed decreased neurogenesis. Treadmill exercise enhanced neurogenesis in the circadian rhythm disturbed mice (Fig. 5).

In the present results, circadian rhythm disruption impaired memory function through inhibiting the expressions of GR and various factors related to neurogenesis and memory consolidation, such as BDNF, p-CREB, p-Erk1/2, PI3K, p-Akt, p-PKC, and Egr-1in the hippocampus. Treadmill exercise effectively improved memory function via enhancing the expressions of GR and above-mentioned factors. Zeitgeber is a cue to reset the internal body clock, such as a change in light or temperature, given by the environment. Treadmill exercise acts as the zeitgeber that improves memory function under the circadian rhythm disrupted conditions.

\section{AUTHOR CONTRIBUTION STATEMENT}

- Full access to all the data in the study and takes responsibility for the integrity of the data and the accuracy of the data analysis: SEK, CJK

- Study concept and design: SEK, KHK

- Acquisition of data: IGK, ESJ, JJJ, LH, SHK

- Analysis and interpretation of data: IGK, ESJ, JJJ, LH, SHK

- Drafting of the manuscript: SEK

- Critical revision of the manuscript for important intellectual content: $C J K$

-Statistical analysis: IGK, ESJ, JJJ, LH, SHK

- Administrative, technical, or material support: $K K, S C$

- Study supervision: $K H K$

\section{REFERENCES}

1. Han DH, Lee YJ, Kim K, Kim CJ, Cho S. Modulation of glucocorticoid receptor induction properties by core circadian clock proteins. Mol Cell Endocrinol 2014;383:170-80.

2. Gerstner JR, Yin JC. Circadian rhythms and memory formation. Nat Rev Neurosci 2010;11:577-88.

3. Hwang DS, Kwak HB, Ko IG, Kim SE, Jin JJ, Ji ES, et al. Treadmill exercise improves memory function depending on circadian rhythm changes in mice. Int Neurourol J 2016;20(Suppl 2):S141-9.

4. Smarr BL, Jennings KJ, Driscoll JR, Kriegsfeld LJ. A time to remember: the role of circadian clocks in learning and memory. Behav Neurosci 2014;128:283-303.

5. Finsterwald C, Alberini CM. Stress and glucocorticoid receptor-dependent mechanisms in long-term memory: from adaptive responses to psychopathologies. Neurobiol Learn Mem 2014;112:17-29.

6. Schoenfeld TJ, Gould E. Stress, stress hormones, and adult neurogenesis. Exp Neurol 2012;233:12-21.

7. Chen DY, Bambah-Mukku D, Pollonini G, Alberini CM. Glucocorticoid receptors recruit the CaMKIIa-BDNF-CREB pathways to mediate memory consolidation. Nat Neurosci 2012;15:1707-14. 8. Tyler WJ, Alonso M, Bramham CR, Pozzo-Miller LD. From acqui- 
sition to consolidation: on the role of brain-derived neurotrophic factor signaling in hippocampal-dependent learning. Learn Mem 2002;9:224-37.

9. Aguiar AS Jr, Castro AA, Moreira EL, Glaser V, Santos AR, Tasca $\mathrm{CI}$, et al. Short bouts of mild-intensity physical exercise improve spatial learning and memory in aging rats: involvement of hippocampal plasticity via AKT, CREB and BDNF signaling. Mech Ageing Dev 2011;132:560-7.

10. Cho JW, Jung SY, Lee SW, Lee SJ, Seo TB, Kim YP, et al. Treadmill exercise ameliorates social isolation-induced depression through neuronal generation in rat pups. J Exerc Rehabil 2017;13:627-33.

11. Kim SE, Ko IG, Shin MS, Kim CJ, Jin BK, Hong HP, et al. Treadmill exercise and wheel exercise enhance expressions of neutrophic factors in the hippocampus of lipopolysaccharide-injected rats. Neurosci Lett 2013;538:54-9.

12. Vivar C, Potter MC, van Praag H. All about running: synaptic plasticity, growth factors and adult hippocampal neurogenesis. Curr Top Behav Neurosci 2013;15:189-210.

13. Pastore S, Hood DA. Endurance training ameliorates the metabolic and performance characteristics of circadian Clock mutant mice. J Appl Physiol 2013;114:1076-84.

14. Son GH, Chung S, Choe HK, Kim HD, Baik SM, Lee H, et al. Adrenal peripheral clock controls the autonomous circadian rhythm of glucocorticoid by causing rhythmic steroid production. Proc Natl Acad Sci U S A 2008;105:20970-5.

15. Jung SY, Kim DY. Treadmill exercise improves motor and memory functions in cerebral palsy rats through activation of PI3K-Akt pathway. J Exerc Rehabil 2017;13:136-42.

16. Sungur AÖ, Stemmler L, Wöhr M, Rust MB. Impaired object recognition but normal social behavior and ultrasonic communication in cofilin1 mutant mice. Front Behav Neurosci 2018;12:25.

17. Jin JJ, Ko IG, Kim SE, Hwang L, Lee MG, Kim DY, et al. Age-dependent differences of treadmill exercise on spatial learning ability between young- and adult-age rats. J Exerc Rehabil 2017;13:381-6.

18. Park HS, Kim TW. Paternal physical exercise improves spatial learning ability by enhancing hippocampal neuroplasticity in male pups born from obese maternal rats. J Exerc Rehabil 2017;13:266-72.

19. Eckel-Mahan KL, Phan T, Han S, Wang H, Chan GC, Scheiner ZS, et al. Circadian oscillation of hippocampal MAPK activity and cAMP: implications for memory persistence. Nat Neurosci 2008; 11:1074-82.

20. Snider KH, Dziema H, Aten S, Loeser J, Norona FE, Hoyt K, et al. Modulation of learning and memory by the targeted deletion of the circadian clock gene Bmall in forebrain circuits. Behav Brain Res 2016;308:222-35.
21. Wardlaw SM, Phan TX, Saraf A, Chen X, Storm DR. Genetic disruption of the core circadian clock impairs hippocampus-dependent memory. Learn Mem 2014;21:417-23.

22. Ortega-Martínez S. A new perspective on the role of the CREB family of transcription factors in memory consolidation via adult hippocampal neurogenesis. Front Mol Neurosci 2015;8:46.

23. Leliavski A, Shostak A, Husse J, Oster H. Impaired glucocorticoid production and response to stress in Arntl-deficient male mice. Endocrinology 2014;155:133-42.

24. Kitchener P, Di Blasi F, Borrelli E, Piazza PV. Differences between brain structures in nuclear translocation and DNA binding of the glucocorticoid receptor during stress and the circadian cycle. Eur J Neurosci 2004;19:1837-46.

25. Suzuki A, Fukushima H, Mukawa T, Toyoda H, Wu LJ, Zhao MG, et al. Upregulation of CREB-mediated transcription enhances both short- and long-term memory. J Neurosci 2011;31:8786-802.

26. Seo TB, Cho HS, Shin MS, Kim CJ, Ji ES, Baek SS. Treadmill exercise improves behavioral outcomes and spatial learning memory through up-regulation of reelin signaling pathway in autistic rats. J Exerc Rehabil 2013;9:220-9.

27. Yoshii A, Constantine-Paton M. Postsynaptic BDNF-TrkB signaling in synapse maturation, plasticity, and disease. Dev Neurobiol 2010;70:304-22.

28. Pascale A, Amadio M, Scapagnini G, Lanni C, Racchi M, Provenzani A, et al. Neuronal ELAV proteins enhance mRNA stability by a PKCadependent pathway. Proc Natl Acad Sci U S A 2005;102:12065-70.

29. Lin TW, Shih YH, Chen SJ, Lien CH, Chang CY, Huang TY, et al. Running exercise delays neurodegeneration in amygdala and hippocampus of Alzheimer's disease (APP/PS1) transgenic mice. Neurobiol Learn Mem 2015;118:189-97.

30. Revest JM, Di Blasi F, Kitchener P, Rougé-Pont F, Desmedt A, Turiault M, et al. The MAPK pathway and Egr-1 mediate stress-related behavioral effects of glucocorticoids. Nat Neurosci 2005;8:664-72.

31. Jones MW, Errington ML, French PJ, Fine A, Bliss TV, Garel S, et al. A requirement for the immediate early gene Zif268 in the expression of late LTP and long-term memories. Nat Neurosci 2001; 4:289-96.

32. Bozon B, Davis S, Laroche S. Regulated transcription of the immediate-early gene Zif268: mechanisms and gene dosage-dependent function in synaptic plasticity and memory formation. Hippocampus 2002;12:570-7.

33. Suri D, Vaidya VA. Glucocorticoid regulation of brain-derived neurotrophic factor: relevance to hippocampal structural and functional plasticity. Neuroscience 2013;239:196-213. 